EDITORIAL

\title{
Core Facility in Medical Research
}

\author{
SK Biswas \\ Department of Biochemistry, Bangabandhu Sheikh Mujib Medical University (BSMMU), Bangladesh
}

Medical research, in this modern era of molecular biology and nano-biotechnology, requires highly sophisticated instruments, very expensive reagents, technical expertise and science-friendly policy in addition to dedicated scientists with innovative ideas. Even the most developed countries sometimes fail to provide adequate funding to support all the required facilities to maintain high-quality research. Many countries have therefore successfully utilized the concept of core facility to upgrade the quality of research and to reduce the cost of research.

Core facility is a centralized collection of highly sophisticated instruments well equipped with necessary reagents and technical staff to support multiple laboratories of an institute or university. It has many benefits for the researchers and for the institutes. A core facility provides specialized services to the internal researchers or laboratories with no service fee or with minimal fee just to cover its running cost, and it does not ask for collaboration or authorship in return. It can also provide services to the external researchers with fee.

Group of laboratories within an institute or university that perform similar types of experiments constitute their own core facility, and as a consequence, there may be multiple core facilities within an institute or university. Harvard Medical School, for example, has many core facilities - flow cytometry facility, conventional electron microscopy facility, molecular electron microscopy facility, molecular genetics core facility, next generation sequencing facility, small molecular NMR facility etc. Each core facility supports innovative research to the respective group of laboratories and also to external laboratories by providing highly specialized equipment and services. A core facility thereby eliminates the need of having every piece of highly expensive instruments in an individual laboratory, and facilitates extensive range of specialized investigations that would otherwise be too expensive for a single laboratory or department. A core facility also eliminates the chance of underutilization of an instrument during its lifespan.

In most cases where it is difficult to buy a highly expensive specialized instrument for a single laboratory due to high cost of procurement and maintenance, a core facility can easily procure and maintain this type of costly instrument and serve several, even many laboratories at a time. For example, every nutritional biochemistry laboratory does not need to have its own atomic absorption spectrophotometer for trace metal analysis. Rather a single atomic absorption spectrophotometer in a core facility can serve many laboratories that investigate trace metals. Similarly, every laboratory that uses genetics and molecular biology techniques does not need to have its own DNA sequencing machine. A molecular biology core facility with a DNAsequencer can provide sequencing facility to many such laboratories at a time. A single animal house that usually serves all the laboratories of an institute or university is also an example of core facility.

Considering the contribution of core facility in medical research especially in poor funding situation it is mandatory to establish medical research core facility in Bangladesh. Institutes, like Bangabandhu Sheikh Mujib Medical University (BSMMU) and Dhaka Medical College, must therefore consider to establish at least one central research core facility to support and upgrade medical research in Bangladesh. This will not only support and encourage internal researchers to perform frontline research but also make the services available to those external researchers who work in smaller institutes. 